\title{
Neoadjuvant Chemotherapy for Patients with Muscle-invasive Urothelial Bladder Cancer Candidates for Curative Surgery: A Prospective Clinical Trial Based on Cisplatin Feasibility
}

\author{
GIOVANNI SCHINZARI ${ }^{1}$, SANTA MONTERISI ${ }^{1}$, FRANCESCO PIERCONTI $^{2}$, GIULIA NAZZICONE $^{3}$, \\ LAURA MARANDINO ${ }^{1}$, ARMANDO ORLANDI ${ }^{1}$, MARCO RACIOPPI $^{4}$, \\ ALESSANDRA CASSANO ${ }^{1}$, PIERFRANCESCO BASSI ${ }^{4}$, CARLO BARONE $^{1}$ and ERNESTO ROSSI ${ }^{1}$ \\ ${ }^{1}$ Medical Oncology, Università Cattolica del Sacro Cuore, Rome, Italy; \\ ${ }^{2}$ Department of Pathology, Università Cattolica del Sacro Cuore, Rome, Italy; \\ ${ }^{3}$ Palliative Care Unit, Hospice Villa Speranza, Rome, Italy; \\ ${ }^{4}$ Department of Urology, Università Cattolica del Sacro Cuore, Rome, Italy
}

\begin{abstract}
Background: Neoadjuvant chemotherapy demonstrated a survival benefit versus cystectomy alone in muscle-invasive urothelial bladder cancer. Despite this advantage, preoperative chemotherapy is not widely employed. When patients are unfit for cisplatin-based regimen, they are often candidates for immediate surgery. In our study, patients with muscle-invasive bladder cancer were treated with neoadjuvant chemotherapy. The principal objective was the rate of complete pathological response $(p C R)$. Secondary end-points were disease-free survival (DFS), overall survival (OS) and toxicity. Patients and Methods: Patients $(n=72)$ with Eastern Cooperative Oncology Group (ECOG) performance status 0-1, clinical stage cT3-4, and/or N+ muscle-invasive bladder cancer were enrolled. The chemotherapy regimen was established according to the cisplatin feasibility. Thirty patients were treated with cisplatin/gemcitabine (Gem) and 42 with carboplatin/Gem. Results: The rate of pCR was 29.2\%, 36\% with cisplatin-based treatment and $23.8 \%$ with carboplatin $(p=0.3574) . D F S$ and $O S$ were longer in $p C R$ patients, while no difference was reported between cisplatin/Gem and Carboplatin/Gem groups. Conclusion: Our results confirm the prognostic value of $p C R$ in neoadjuvant chemotherapy for muscle-invasive bladder cancer. When the patients are not fit for cisplatin, a carboplatin/Gem regimen represents a valid
\end{abstract}

Correspondence to: Giovanni Schinzari, Medical Oncology, Università Cattolica del Sacro Cuore, Largo F. Vito 1, 00168, Rome, Italy. Tel: +390630156318, Fax: +390630156733, e-mail: Giovanni.schinzari@unicatt.it

Key Words: Cisplatin, carboplatin, neoadjuvant, urothelial bladder cancer, pathological response. option because of comparable long-term outcome. When cisplatin is not feasible, the exclusion of a preoperative treatment is not justified.

Muscle invasive bladder cancer is one of the most aggressive tumor. In the United States, it accounts for 4.4 deaths $\mathrm{x}$ 100,000 people per year (1). Despite the local control with radical cystectomy and pelvic lymphadenectomy, about $40 \%$ of patients relapse within 5 years from surgery (2). The presence of micrometastases, not clinically detectable at diagnosis, is the major cause of recurrence (3). Indeed, neoadjuvant chemotherapy, which has been explored in several trials, was introduced with the aim to treat subclinical disease. A large phase III study showed a longer survival for patients receiving the combination of methotrexate, vinblastine, doxorubicin and cisplatin (M-VAC) before surgery compared with patients treated with cystectomy alone (4). Two meta-analyses demonstrated a 5\% overall benefit at five years for platinum-based pre-operative treatment versus cystectomy alone $(5,6)$. The efficacy of neoadjuvant chemotherapy is associated with primary tumor downstaging, which is considered a potential surrogate for survival (7-9). Despite the advantages, the preoperative treatment is not yet largely employed due to concerns about delaying curative surgery for chemotherapy side effects and increasing surgical complications (10). Furthermore, several studies on neoadjuvant chemotherapy included cT2N0 patients (4-14), with a low risk of recurrence only with radical cystectomy (15). Chemotherapy could be an overtreatment for this stage of disease. Surgery is also preferred to chemotherapy when patients are unfit for Cisplatin-based treatment. Indeed, Cisplatin infeasibility is considered a negative prognostic factor for outcome of neoadjuvant chemotherapy (16). Nevertheless, data also with the use of carboplatin in 
neoadjuvant setting have been reported (17-19), however a comparison between cisplatin and carboplatin is not available.

In the present phase II prospective study we used criteria of cisplatin feasibility (16) to select patients for cisplatin/ gemcitabine (Gem) or carboplatin/Gem as neoadjuvant chemotherapy for bladder cancer. This study aimed to confirm the effectiveness of carboplatin in neoadjuvant setting and to compare the outcomes of the two treatments (cisplatin/Gem or carboplatin/Gem). These data could be useful to define the best strategy for muscle-invasive bladder cancer according to the clinical characteristics of the patients.

\section{Patients and Methods}

Patients with muscle-invasive bladder cancer and radiological evidence of clinical T3/T4 and/or clinical node positive disease were included. All patients were candidates for surgical treatment with curative intent. Diagnosis of muscle-invasive urothelial cancer was obtained through specimen of transurethral resection of bladder (TURB). Clinical staging was completed with a lung and abdomen computed tomography (CT) scan and bone scan. Patients with distant metastases were excluded. Other inclusion criteria were: Eastern Cooperative Oncology Group (ECOG) Performance Status less than 2, adequate bone marrow, and liver function. Written informed consent was required. Patients who have received prior chemo- or radiotherapy for any cause were excluded. Prior intravescical therapy was allowed if it has been completed more than 3 months before starting chemotherapy. Treatment options were gemcitabine $1,000 \mathrm{mg} / \mathrm{m}^{2}$, on days 1 and 8 , plus cisplatin $80 \mathrm{mg} / \mathrm{m}^{2}$, on day 1 , or carboplatin to an area under the curve (AUC) of 5 , on day 1 . Cycles were repeated every 21 days. Carboplatin was used for monorenal patients or in presence of chronic renal failure (creatinine clearance $<50 \mathrm{ml} / \mathrm{min}$ ) or secondary hydronephrosis (even if corrected) or cardiac dysfunction with ejection fraction (EF) $<50 \%$ or respiratory failure (Table I). Dose adjustments or cycles delays were introduced in case of G3/4 toxicity according to Common Toxicity Criteria (CTC v4.0). After 3 cycles of chemotherapy, lung and abdominal CT scan was repeated. Patients without distant progressive disease underwent radical cystectomy with different urinary diversions (orthtotopic neobladder or Bricker ileal conduit).

The primary endpoint was the rate of complete pathological response (pCR) for Cisplatin/Gem neoadjuvant chemotherapy or Carboplatin/Gem. Pathological stage was evaluated on radical cystectomy specimens and compared with baseline clinical stage on primary tumor and nodes. A complete pathological response was defined as the absence of invasive residual tumor in bladder and nodes (pT0N0 or pTisN0 stage).

Secondary endpoints were disease-free survival (DFS), overall survival (OS) and toxicity both for Cisplatin and Carboplatin group of patients. DFS has been defined as the time from day 1 of systemic treatment to disease progression or death from any cause, while OS as the time from day 1 of chemotherapy to death from any cause.

Statistical analysis. DFS and OS were estimated by the KaplanMeier method using the log-rank test for subgroups comparison. Cox-proportional hazard model with $95 \%$ confidence interval (CI) was used to calculate the hazard ratios (HR). Chi-squared test was used to evaluate the association of categorical data. All $p$-values $\leq 0.05$ were considered statistically significant.
Table I. Criteria for cisplatin infeasibility (one or more).

Criteria for cisplatin infeasibility

Monorenal condition

Creatinine Clearance $<50 \mathrm{ml} / \mathrm{min}$

Secondary Hydronephrosis

Cardiac dysfunction with $\mathrm{EF}<50 \%$

Respiratory failure

EF, Ejection fraction.

Table II. Patient characteristics and therapeutic details.

\begin{tabular}{lc}
\hline Demographic and clinical characteristics of patients & \\
\hline Median age (range) & 66.6 y $(44-82)$ \\
M/F & $64 / 8$ \\
Smoker (Y/N) & $58 / 14$ \\
Prior intravescical therapy (Y/N) & $45 / 27$ \\
Clinical staging & \\
cT3N0 & 33 \\
cT4N0 & 21 \\
any T cN+ & 18 \\
Cisplatin/Gem & 30 \\
Carboplatin/Gem & 42 \\
Orthotopic neobladder & 18 \\
Bricker ileal conduit & 54 \\
\hline
\end{tabular}

Y, Years; M, male; F, female; Y/N, yes/no; Gem, gemcitabine.

\section{Results}

From October 2010 to January 2015, 72 patients were enrolled and were followed-up for at least 30 months at our institution. Median age was 66.6 years (range 44-82 years); 28 patients were more than 70 years old. Thirty patients (41.7\%) were treated with Cisplatin/Gem, while $42(58.3 \%)$ with Carboplatin/Gem. Patient characteristics and therapeutic details are summarized in Table II.

No patient progressed with distant metastases after chemotherapy. All patients underwent surgery after a median of 32 days after the last chemotherapy course.

Twenty-one patients achieved pCR (29.2\%), 10/42 (23.8\%) treated with Carboplatin and 11/30 (36\%) with Cisplatin. The different pCR rate observed between Cisplatin- and Carboplatin-based treatment was not significant $(p=0.35)$. Response to the treatments is summarized in Table III.

Median OS of the whole population was 47.6 months, while the DFS was 32 months (Figure 1).

Median OS of the patients achieved pCR was not reached, whereas median OS of the patients with residual tumor was 46 months (Figure 2A). A correlation between complete 
Table III. Complete response to treatment.

\begin{tabular}{lc}
\hline & $\mathrm{pCR}$ \\
\hline All population & $21(29.2 \%)$ \\
Cisplatin & $11(36 \%)$ \\
Carboplatin & $10(23.8 \%)$ \\
& $p=0.3574 *$ \\
\hline
\end{tabular}

*Chi-squared test; pCR, complete pathological response.

pathological response and OS was found $(p=0.001$ $\mathrm{HR}=0.0768,95 \% \mathrm{CI}=0.0338-0.1747)$. DFS of the patients with pCR was not reached, whereas DFS for the patients with residual tumor was 17.5 months $(p=0.0001 ; \mathrm{HR}=0.1049,95 \%$ $\mathrm{CI}=0.0523-0.2102$ ) (Figure 2B). The treatment with Cisplatin or Carboplatin did not influence the median OS $(p=0.89$; $\mathrm{HR}=1.0584,95 \% \mathrm{CI}=0.4706-2.3804$ ) (Figure 3A). Similarly, DFS was not influenced by the platinum compound ( $p=0.5738$; $\mathrm{HR}=1.2220,95 \% \mathrm{CI}=0.6153-2.4270$ ) (Figure 3B).

Toxicity is reported in Table IV. Grade 3-4 neutropenia was observed in $5(11.9 \%)$ patients treated with Carboplatin and $2(6.6 \%)$ patients treated with cisplatin. Grade 3-4 anemia occurred in $1(1.3 \%)$ patient treated with Carboplatin and 2 $(6.6 \%)$ patients treated with cisplatin. Grade 3-4 thrombocytopenia was reported in $4(9.5 \%)$ patients with carboplatin and in $1(3.3 \%)$ patient with cisplatin. One patient in carboplatin/Gem group discontinued the chemotherapy after 2 cycles because of haematological toxicity. Grade 3 nausea and vomiting was observed in 2 patients $(6.6 \%)$ with cisplatin. Grade 1-2 peripheral neuropathy was experienced by 3 patients with Cisplatin. No treatment-related death occurred and there was not reported any increase of postoperative morbidity in comparison to historical controls. One patient died for post-surgical complications.

\section{Discussion}

During the last years, there were not observed any impressive improvements in the treatment of muscle-invasive bladder cancer. Except for Vinflunine, which demonstrated a modest benefit as second-line therapy in advanced disease, no other agent has been recently approved (20). Therefore, the optimal use of the available drugs, the definition of an effective strategy and the choice of the best therapeutic sequence could be crucial.

Neoadjuvant therapy is an effective strategy to improve the outcome. Several studies demonstrated a survival advantage (4-6), which is remarkable when the pCR is achieved (7-9). During the last years, neoadjuvant treatment has become more common (21). Nevertheless, it is not always proposed
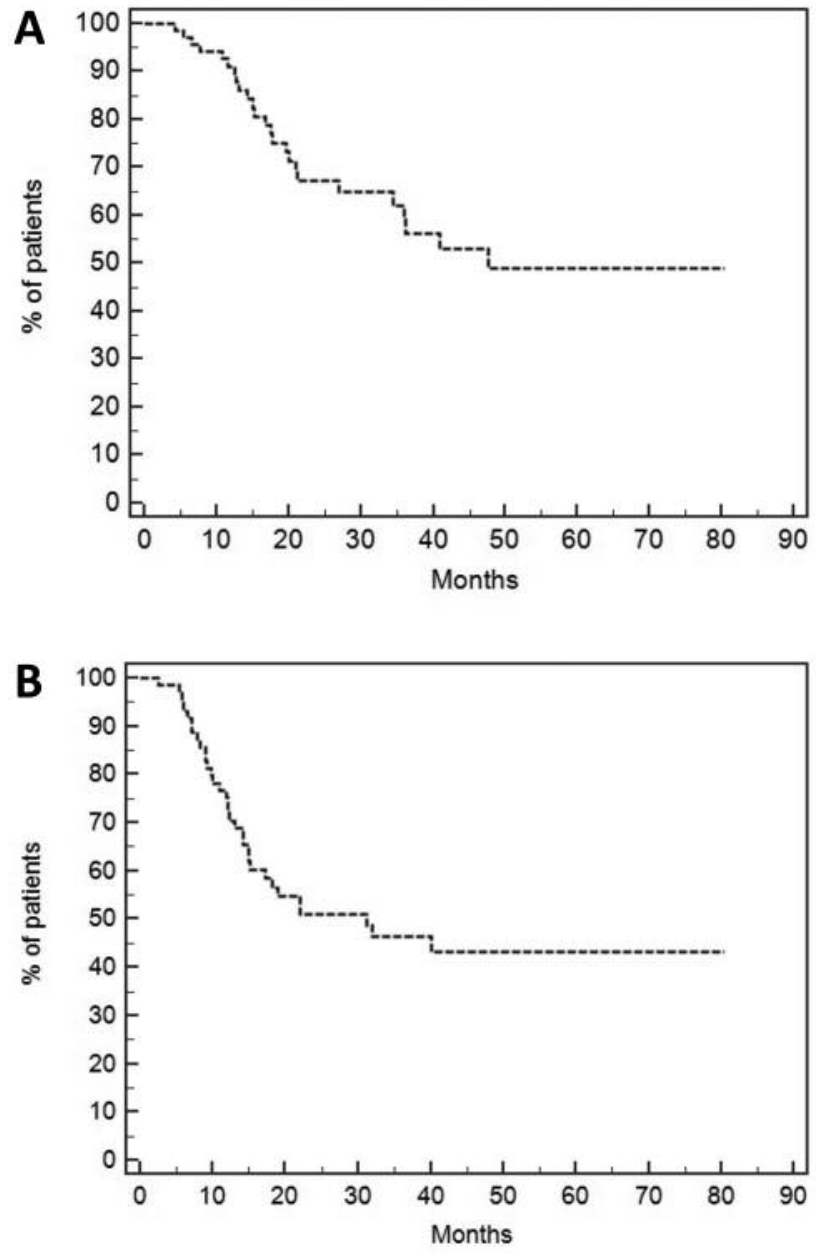

Figure 1. (A) Overall survival (47.6 months) and (B) disease-free survival (32 months) of the entire population.

to all the patients with muscle-invasive bladder cancer (10), particularly when patients are considered unfit for cisplatin (16). In this study, a platinum-Gem chemotherapy was offered as neoadjuvant treatment for muscle-invasive bladder cancer. Contrarily to many other studies, patients with cT2 stage were not included because of the low risk of recurrence after the cystectomy alone. The choice of cisplatin or carboplatin was based on defined criteria. Carboplatin was preferred to cisplatin when patients had one of the following clinical characteristics: monorenal condition, creatinine clearance $<50 \mathrm{ml} / \mathrm{min}$, secondary hydronephrosis, cardiac dysfunction with $\mathrm{EF}<50 \%$, respiratory failure. Afterwards, we evaluated the outcome of the two groups of patients (carboplatin/Gem and cisplatin/Gem). In our study, median OS of the whole population was 47.6 months, while the DFS 32 months. Twenty-one patients (29.2\%) obtained a pCR, 
A

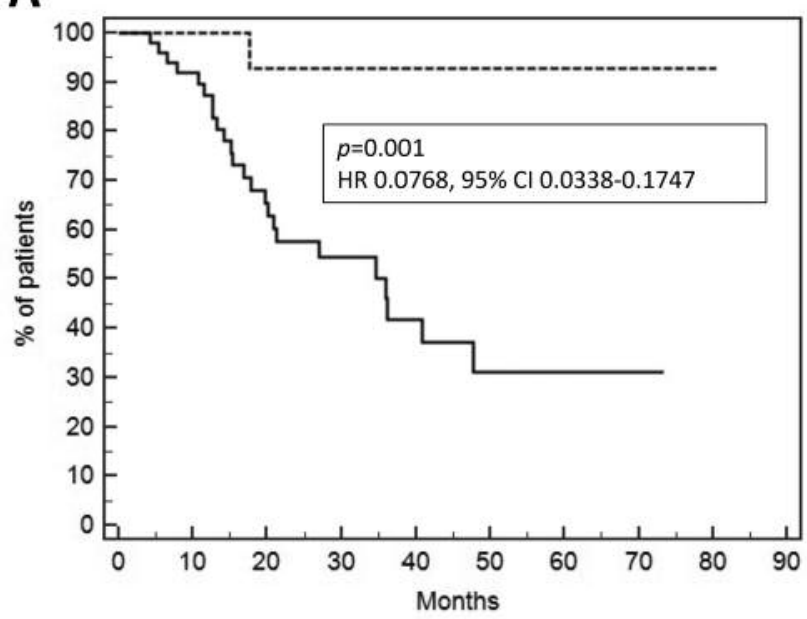

B

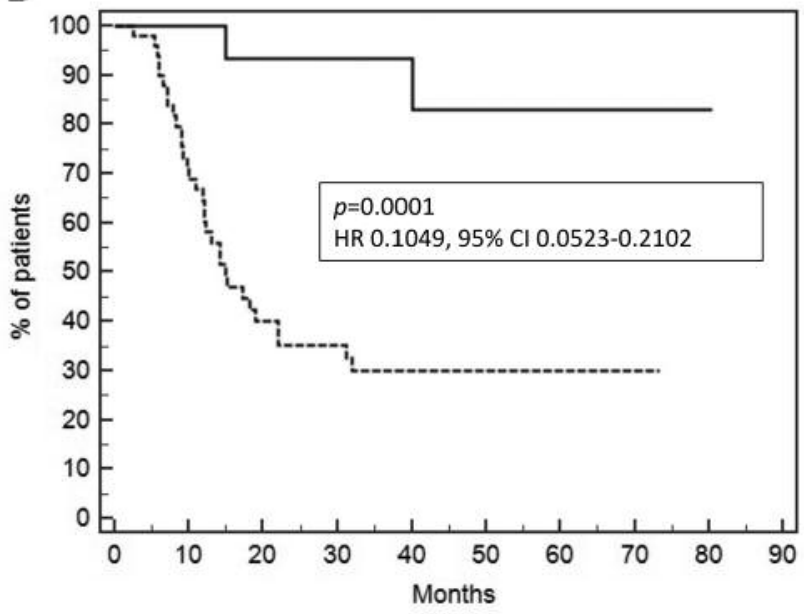

Figure 2. (A) Overall survival and $(B)$ disease-free survival of patients achieving a complete pathological response (dotted line) or with residual tumor (solid line).

which was correlated with prolonged OS and DFS. These results are particularly relevant considering the exclusion of cT2 patients, who could have influenced the analysis because of their better prognosis. These consistent data are similar to those reported in previous papers on neoadjuvant therapy for muscle-invasive bladder cancer and confirm the effectiveness of this strategy. In fact, the study by Galsky and colleagues has demonstrated $29 \%$ and $31 \%$ pCR rate with methotrexate, vinblastine, doxorubicin plus cisplatin combination and cisplatin/Gem, respectively, without significant difference between the two regimens (22). Additionally, the pooled analysis by Yuh et al. showed a $25.6 \%$ pCR rate with the combination of Cisplatin and Gemcitabine (7), which was correlated with a longer survival. Interestingly, in our study the percentage of $\mathrm{pCR}$ for the patients treated with Cisplatin and carboplatin was comparable $(p=0.35)$. Moreover, the
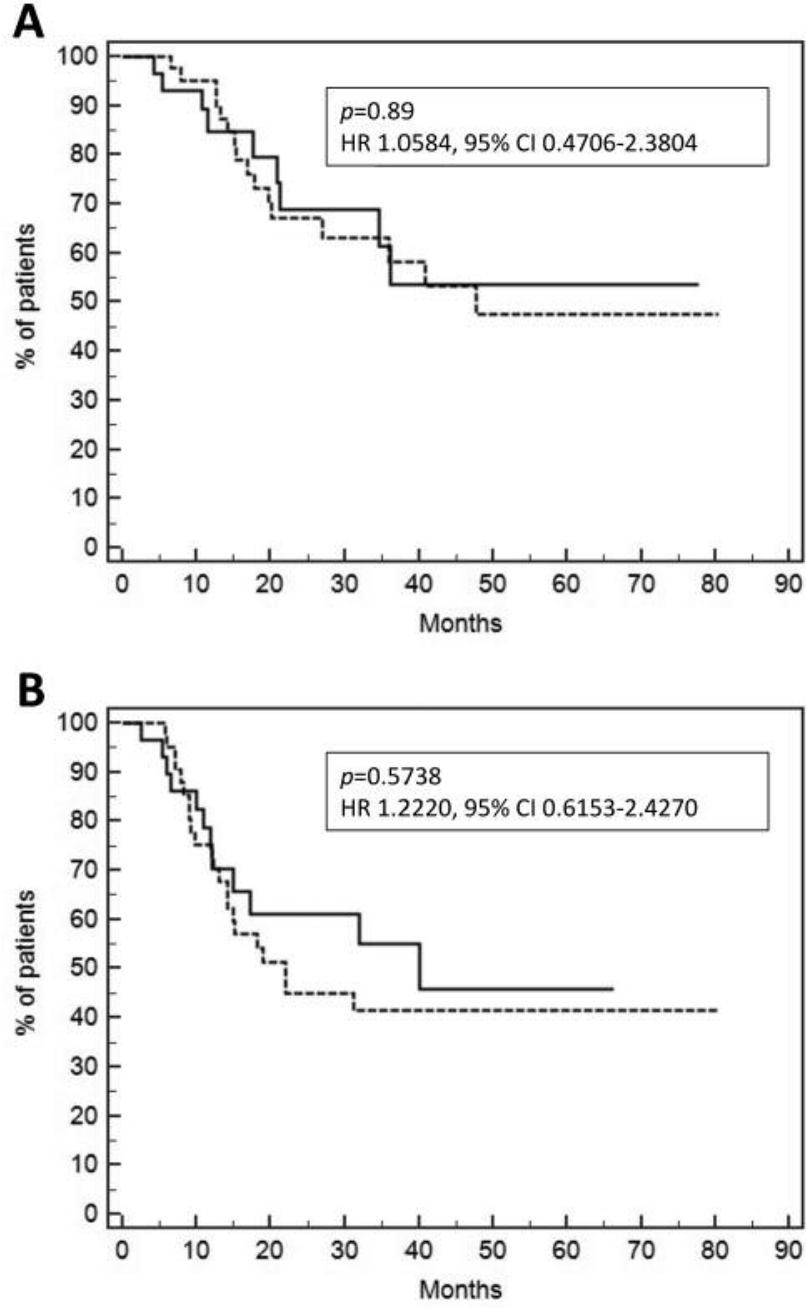

Figure 3. (A) Overall survival and (B) disease-free survival of patients treated with cisplatin (solid line) or carboplatin (dotted line).

platinum compound - cisplatin or carboplatin - did not influence the survival benefit, both in terms of DFS and OS. Our results demonstrated that the platinum-feasibility criteria could be very useful to identify patients with muscle-invasive bladder cancer who are candidates for cisplatin or carboplatin treatment. Patients selected up-front to receive cisplatin/Gem or carboplatin/Gem according to the clinical parameters of cisplatin feasibility could obtain comparable results by a neoadjuvant therapy. The different platinum-compounds did not show any substantial differences neither in terms of toxicity. Grade 3-4 haematological and non-haematological toxicity was mild, probably due to the limited number of chemotherapy cycles administered. In the carboplatin group G3-4 neutropenia and thrombocytopenia were slightly more frequent than in cisplatin group, in which we observed more cases of anemia and peripheral neuropathy. Neoadjuvant 
Table IV. Toxicity of the chemotherapy regimens.

\begin{tabular}{|c|c|c|c|c|}
\hline & \multicolumn{2}{|c|}{ Cisplatin/Gem } & \multicolumn{2}{|c|}{ Carboplatin/Gem } \\
\hline & $\begin{array}{l}\text { Grade } 1-2 \\
\text { N.pts }(\% *)\end{array}$ & $\begin{array}{l}\text { Grade } 3-4 \\
\text { N. pts }(\% *)\end{array}$ & $\begin{array}{c}\text { Grade } 1-2 \\
\text { N. pts }(\% * *)\end{array}$ & $\begin{array}{c}\text { Grade } 3-4 \\
\text { N. pts }(\% * *)\end{array}$ \\
\hline Anemia & $7(23,3)$ & $2(6,6)$ & $2(4,7)$ & $1(1,3)$ \\
\hline Thrombocytopenia & $4(13,3)$ & $1(3,3)$ & $7(16.6)$ & $4(9,5)$ \\
\hline Neutropenia & $5(16,6)$ & $2(6,6)$ & $11(26,1)$ & $5(11,9)$ \\
\hline Asthenia & $8(24)$ & - & $3(7,1)$ & - \\
\hline Nausea-Vomiting & $12(40)$ & $2(6,6)$ & $7(16,6)$ & - \\
\hline Diarrhoea & - & - & $1(2,4)$ & - \\
\hline Stomatitis & $1(3,3)$ & - & $1(2,3)$ & - \\
\hline Neurotoxicity & $3(10)$ & - & - & - \\
\hline Kindey injury & $1(3,3)$ & - & - & - \\
\hline AST/ALT elevation & - & - & - & - \\
\hline LVEF decreasing & - & - & - & - \\
\hline
\end{tabular}

*Calculated on 30 cisplatin/Gem patients; **Calculated on 42 carboplatin/Gem patients. Gem, gemcitabine; N. pts, number of patients, AST, aspartate aminotransferase; ALT, alanine aminotransferase; LVEF, left ventricular ejection fraction.

therapy was feasible and effective, also considering the high rate of elderly patients (about $40 \%$ ) in the study population.

A short course of neoadjuvant chemotherapy was associated to a low incidence of serious adverse events and did not increase the post-operative morbidity. A remarkable result of the present study is the comparable efficacy and toxicity of cisplatin and carboplatin when used in patients selected by well-defined clinical parameters. The next goal of future investigations is the identification of predictive factors, which could allow to increase the pCR rate, addressing immediate surgery when chemotherapy cannot be effective.

In conclusion, our data support the neoadjuvant treatment for muscle-invasive bladder cancer, encouraging the use of carboplatin for the patients that cannot receive cisplatin.

\section{References}

1 Ferlay J, Soerjomataram I, Ervik M, Dikshit R, Eser S, Mathers C, Rebelo M, Parkin DM, Forman D and Bray, F: GLOBOCAN 2012 v1.0, Cancer incidence and mortality worldwide: IARC CancerBase No. 11 (Internet). Lyon, France: International agency for research on cancer; 2013. Available from: http://globocan.iarc.fr, accessed on 16/Sep/2017.

2 Kim B, Choi HJ, Kim MH and Cho KS: Recurrence patterns of bladder transitional cell carcinoma after radical cystectomy. Acta Radiol 53: 943-949, 2012.

3 Kurahashi T, Hara I, Oka N, Kamidono S, Eto H, Miyake H: Detection of micrometastases in pelvic lymph nodes in patients undergoing radical cystectomy for locally invasive bladder cancer by real-time reverse transcriptase-PCR for cytokeratin 19 and uroplakin II. Clin Cancer Res 11: 3773-3777, 2005.

4 Grossman HB, Natale RB, Tangen CM, Speights VO, Vogelzang NJ, Trump DL, deVere White RW, Sarosdy MF, Wood DP Jr., Raghavan D and Crawford ED: Neoadjuvant chemotherapy plus cystectomy compared with cystectomy alone for locally advanced bladder cancer. N Engl J Med 349: 859-866, 2003.

5 Advanced Bladder Cancer (ABC) Meta-analysis Collaboration: Neoadjuvant chemotherapy in invasive bladder cancer: a systematic review and meta-analysis. Lancet 361: 1927-1934, 2003.

6 Advanced Bladder Cancer (ABC) Meta-analysis Collaboration: Neoadjuvant chemotherapy in invasive bladder cancer: update of a systematic review and meta-analysis of individual patient data advanced bladder cancer (ABC) meta-analysis collaboration. Eur Urol 48: 202-205, 2005.

7 Yuh BE, Ruel N, Wilson TG, Vogelzang N and Pal SK: Pooled analysis of clinical outcomes with neoadjuvant cisplatin and gemcitabine chemotherapy for muscle invasive bladder cancer. J Urol 189: 1682-1686, 2013.

8 Sonpavde G, Goldman BH, Speights VO, Lerner SP, Wood DP, Vogelzang NJ, Trump DL, Natale RB, Grossman HB and Crawford ED: Quality of pathologic response and surgery correlate with survival for patients with completely resected bladder cancer after neoadjuvant chemotherapy. Cancer 115: 4104-4109, 2009.

9 Scattoni V, Bolognesi A, Cozzarini C, Francesca F, Grasso M, Galli L, Torelli T, Campo B, Villa E and Rigatti P: Neoadjuvant CMV chemotherapy plus radical cystectomy in locally advanced bladder cancer: the impact of pathologic response on long-term results. Tumori 82: 463-469, 1996.

10 Zaid HB, Patel SG, Stimson CJ, Resnick MJ, Cookson MS, Barocas DA and Chang SS: Trends in the utilization of neoadjuvant chemotherapy in muscle-invasive bladder cancer: results from the National Cancer Database. Urology 83: 75-80, 2014.

11 Alva AS, Tallman CT, He C, Hussain MH, Hafez K, Montie JE, Smith DC, Weizer AZ, Wood D and Lee CT: Efficient delivery of radical cystectomy after neoadjuvant chemotherapy for muscle-invasive bladder cancer: a multidisciplinary approach. Cancer 118: 44-53, 2012. 
12 Scosyrev E, Messing EM, van Wijngaarden E, Peterson DR, Sahasrabudhe D, Golijanin D and Fisher SG: Neoadjuvant gemcitabine and cisplatin chemotherapy for locally advanced urothelial cancer of the bladder. Cancer 118: 72-81, 2012.

13 Dreicer R, Kollmorgen TA, Smith RF and Williams RD: Neoadjuvant cisplatin, methotrexate and vinblastine for muscleinvasive bladder cancer: long-term follow-up. J Urol 150: 849$852,1993$.

14 Stein JP and Skinner DG: The role of lymphadenectomy in highgrade invasive bladder cancer. Urol Clin North Am 32: 187-197, 2005.

15 Ghoneim MA, el-Mekresh MM, el-Baz MA, el-Attar IA and Ashamallah A: Radical cystectomy for carcinoma of the bladder: critical evaluation of the results in 1,026 cases. J Urol 158: 393399, 1997.

16 Niegisch G and Albers P: Which patients benefit the most from neoadjuvant chemotherapy in advanced bladder cancer? Curr Opin Urol 21: 434-439, 2011.

17 Murasawa H, Koie T, Ohyama C, Yamamoto H, Imai A, Hatakeyama S, Yoneyama T, Hashimoto Y, Iwabuchi I, Ogasawara $\mathrm{M}$ and Kawaguchi T: The utility of neoadjuvant gemcitabine plus carboplatin followed by immediate radical cystectomy in patients with muscle-invasive bladder cancer who are ineligible for cisplatin-based chemotherapy. Int J Clin Oncol 22: 159-165, 2017.

18 Koie T1, Ohyama C, Hashimoto Y, Hatakeyama S, Yamamoto H, Yoneyama $\mathrm{T}$ and Kamimura $\mathrm{N}$ : Efficacies and safety of neoadjuvant gemcitabine plus carboplatin followed by immediate cystectomy in patients with muscle-invasive bladder cancer, including those unfit for cisplatin: a prospective single-arm study. Int J Clin Oncol 18: 724-730, 2013.
19 Koie T, Ohyama C, Yamamoto H, Imai A, Hatakeyama S, Yoneyama T, Hashimoto Y, Yoneyama $\mathrm{T}$ and Tobisawa $\mathrm{Y}$ : Neoadjuvant gemcitabine and carboplatin followed by immediate cystectomy may be associated with a survival benefit in patients with clinical T2 bladder cancer. Med Oncol 31: 949-954, 2014.

20 Bellmunt J, Fougeray R, Rosenberg JE, von der Maase H, Schutz FA, Salhi Y, Culine S and Choueiri TK: Long term survival results of a randomized phase III trial of vinflunine plus best supportive versus best supportive care alone in advanced urothelial carcinoma patients after failure of platinum-based chemotherapy. Ann Oncol 24: 1466-1472, 2013.

21 Krabbe LM1, Westerman ME, Margulis V, Raj GV, Sagalowsky AI, Courtney K, Arriaga Y and Lotan Y: Changing trends in utilization of neoadjuvant chemotherapy in muscle-invasive bladder cancer. Can J Urol 22: 7865-7875, 2015.

22 Galsky MD, Pal SK, Chowdhury S, Harshman LC, Crabb SJ, Wong YN, Yu EY, Powles T, Moshier EL, Ladoire S, Hussain SA, Agarwal N, Vaishampayan UN, Recine F, Berthold D, Necchi A, Theodore C, Milowsky MI, Bellmunt J and Rosenberg JE: Comparative effectiveness of gemcitabine plus cisplatin versus methotrexate, vinblastine, doxorubicin, plus cisplatin as neoadjuvant therapy for muscle-invasive bladder cancer. Cancer 121: 2586-2593, 2015. 